TUM-HEP-940/14

OUTP-14-07P

April 24, 2014

\title{
Muon anomalous magnetic moment and penguin loops in warped extra dimensions*
}

\author{
M. Beneke, P. Moch \\ ${ }^{a}$ Physik Department T31, Technische Universität München, \\ James-Franck-Straße 1, D - 85748 Garching, Germany \\ J. ROHRWILD \\ Rudolf Peierls Centre for Theoretical Physics, University of Oxford, \\ 1 Keble Road, Oxford OX1 3NP, United Kingdom
}

\begin{abstract}
We describe the computation of the one-loop muon anomalous magnetic moment and radiative penguin transitions in the minimal and custodially protected RandallSundrum model. A fully five-dimensional (5D) framework is employed to match the $5 \mathrm{D}$ theory onto the Standard Model extended by dimension-six operators. The additional contribution to the anomalous magnetic moment from the gauge-boson exchange contributions is

$$
\Delta a_{\mu} \approx 8.8(27.2) \cdot 10^{-11} \times(1 \mathrm{TeV} / T)^{2},
$$

where the first (second) number refers to the minimal (custodially-protected) model. Here $1 / T$ denotes the location of the $\mathrm{TeV}$ brane in conformal coordinates, and is related to the mass of the lowest gauge-boson $\mathrm{KK}$ excitation by $M_{\mathrm{KK}} \approx 2.35 T$. We also determine the Higgs-exchange contribution, which depends on the 5D Yukawa structure and the precise interpretation of the localisation of the Higgs field near or at the $\mathrm{TeV}$ brane.
\end{abstract}

\footnotetext{
*To appear in: Proceedings of the international conference on "Flavor Physics and Mass Generation", Nanyang Technological University, Singapore, 10 - 14 February 2014.
} 


\section{Introduction}

The Randall-Sundrum model ${ }^{1}$ with bulk Standard Model (SM) fields and brane-localized Higgs field offers a simultaneous solution to the gauge-gravity and flavour hierarchy problems of the SM at the price of introducing an additional curved space dimension, which would manifest itself through a discrete spectrum of Kaluza-Klein (KK) resonances, possibly visible at the Large Hadron Collider (LHC). Its phenomenology has been studied in very much detail, but mostly at tree level. Some of the strongest constraints on the SM arise, however, from effects that exist only at the loop level. Important examples are the anomalous magnetic and electric dipole moments, lepton-flavour violation and quark flavour-changing neutral current processes related to the radiative, chirality-violating ("penguin") amplitude $f_{i} \rightarrow f_{j} \gamma$. Higgs production and decay is a more recent addition to the list of loop-induced phenomena of interest. These processes are now also being studied in the Randall-Sundrum model (Higgs production and decay ${ }^{2-8}$ lepton $^{9,10}$ and quark flavour violation $\left.{ }^{11-13}\right)$. In this article we describe the computation of one-loop radiative penguin transitions, and the muon anomalous magnetic moment in particular, in a fully five-dimensional (5D) framework following Refs., ${ }^{10,14}$ to which we refer for the more technical details.

Observables related to flavour often provide very high lower limits on the value of the lowest KK resonance, which can however be avoided, if the 5D Yukawa matrices are not generic (anarchic). For example, in a generic setting, the absence of non-standard CP violation in kaon mixing requires the first KK gluon to be heavier than $20 \mathrm{TeV},{ }^{15,16}$ far beyond the reach of the LHC. The modification of Higgs production and signal strengths also depend on the unknown 5D Yukawa couplings. More model-parameter independent limits follow from electroweak precision observables, especially the $S$-parameter (since the $T$-parameter depends on whether the model is endowed with custodial symmetry), and of course the non-observation of direct production of KK resonances at the LHC. These push the KK scale into the multi-TeV range.

The anomalous magnetic moment is interesting in several respects. First, it is very precisely measured (and in some tension with the SM prediction). Second, it receives contributions from gauge-boson and Higgs exchange in the loop. As we discuss below, the former are insensitive to the 5D Yukawa couplings and fall into the category of "electroweak precision tests". The Higgs contributions, on the other hand, are model-parameter-dependent similar to flavour and Higgs observables. Moreover, they are sensitive to the scale set by the localization of the Higgs boson near the $\mathrm{TeV}$ brane in the Randall-Sundrum space-time, an issue that is also important for the interpretation of Higgs production. ${ }^{5,6}$ We discuss the subtleties associated with this issue in the framework of a 5D calculation in the RS theory with unbroken electroweak symmetry. Last but not least, the anomalous magnetic moment in the RS model is a non-trivial but instructive case for setting up and performing loop calculations in a quantum field theory in curved space-time with boundaries.

\section{From the 5D bulk to a $4 \mathrm{D}$ effective Lagrangian}

The RS space-time consists of a slice of Anti-de-Sitter space limited by four-dimensional flat branes. In conformal coordinates the metric of the $5 \mathrm{D}$ bulk is

$$
d s^{2}=\left(\frac{1}{k z}\right)^{2}\left(\eta_{\mu \nu} d x^{\mu} d x^{\nu}-d z^{2}\right)
$$


where $k \sim M_{\mathrm{Pl}} \sim 10^{19} \mathrm{GeV}$ is of order of the Planck scale $M_{\mathrm{Pl}}$, while the four-dimensional boundaries are located at $z=1 / k$ and $z=1 / T$ with $T$ of order $\mathrm{TeV}$. The explicit appearance of a $\mathrm{TeV}$ scale parameter is a coordinate artifact. The proper distance between the two branes, $1 / k \times \ln (k / T)$, is naturally only a few times the Planck length when $T$ varies over many orders of magnitude.

Since the present non-observation of KK resonances requires the scale $T$ to be much larger than the scale of the SM, set by the Higgs vacuum expectation value $v$, the only dynamical degrees of freedom below the scale $T$ are the KK zero modes, which are associated with the usual SM fields. We can therefore match the RS theory onto an effective four-dimensional theory at the scale $\mu\left(T \gg \mu \gg M_{\mathrm{EW}}\right)$, whose Lagrangian consists of the SM Lagrangian plus $\mathrm{SU}(3) \times \mathrm{SU}(2) \times \mathrm{U}(1)$ invariant higher-dimension operators built from $\mathrm{SM}$ fields:

$$
\mathcal{L}_{\mathrm{RS}}^{(5 \mathrm{D})} \quad \longrightarrow \quad \mathcal{L}_{\text {eff }}=\mathcal{L}_{\mathrm{SM}}+\frac{1}{T^{2}} \sum_{i} c_{i} \mathcal{O}_{i}
$$

The dominant effects are captured by dimension-six operators. ${ }^{17,18}$ Since the matching coefficients $c_{i}$ are dominated by distances $\lesssim 1 / T$, the Higgs bilinear term in $V(\Phi)$ can be treated as a perturbation, and the $c_{i}$ can be computed in the theory with unbroken electroweak gauge symmetry. This results in a great technical simplification for the 5D propagators of the gauge and fermion fields.

We note that the 5D theory is non-renormalizable and must itself be defined as an effective theory below a scale $\Lambda$ that should be at least a few times the Planck scale. It is generally assumed (and required to solve the hierarchy problem) that in the mixed representation the four-dimensional loop momenta should be cut-off at a value $\Lambda(z)$ that depends on the position $z$ in the fifth dimension. If $\Lambda(1 / k)$ is a few times the Planck scale, then the cut-off $\Lambda(1 / T)$ relevant to processes dominated by physics near the $\mathrm{TeV}$ brane should be a few times the $\mathrm{TeV}$ scale. This appears to be in conflict with the $5 \mathrm{D}$ formalism, which encodes the sum over all KK states rather than including only the few below the cut-off $\Lambda(1 / T)$. However, for a finite quantity such as the anomalous magnetic moment of the muon, the KK sum must converge, and the effect of including the entire tower relative to the truncation is of order $T^{2} / \Lambda(1 / T)^{2}$, which is the generic size of corrections expected from the UV completion of the RS model. Besides, the general framework of renormalization in curved space-time should apply to the RS theory treated as a 5D quantum field theory, and makes no reference to the KK decomposition.

The matching strategy outlined above does not depend on the details of the 5D Lagrangian though the matching coefficients $c_{i}$ do. We will use the simplest set-up of the Randall-Sundrum model. All SM fields are allowed to propagate throughout the five-dimensional bulk, except for the Higgs doublet which is confined to the IR brane at $z=1 / T$. No further field content is added. Quarks as well as the strong sector are not relevant for the following discussion of leptonic transitions. ${ }^{\mathrm{a}}$ This "minimal" model is no longer attractive from the phenomenological point of view, since tree-level custodial-symmetry violation requires the scale $T$ to be larger than roughly $4 \mathrm{TeV} \cdot{ }^{19-21} \mathrm{It}$ is, however, useful to illustrate the general approach. At the end of this article, we discuss the extension of the computation to the RS model with custodial symmetry.

Only a few dimension-six operators from the general expression (2) are relevant to the

${ }^{\text {a }}$ The Lagrangian is specified in detail in Ref. ${ }^{10}$ 
leptonic radiative transitions at the one-loop level:

$$
\begin{aligned}
\sum_{i} c_{i} \mathcal{O}_{i}= & a_{B, i j} \bar{L}_{i} \Phi \sigma_{\mu \nu} E_{j} B^{\mu \nu}+a_{W, i j} \bar{L}_{i} \tau^{a} \Phi \sigma_{\mu \nu} E_{j} W^{a, \mu \nu}+\text { h.c. } \\
& +b_{i j}\left(\bar{L}_{i} \gamma^{\mu} L_{i}\right)\left(\bar{E}_{j} \gamma_{\mu} E_{j}\right)+c_{1, i}\left(\bar{E}_{i} \gamma_{\mu} E_{i}\right)\left(\Phi^{\dagger} i D^{\mu} \Phi\right) \\
& +c_{2, i}\left(\bar{L}_{i} \gamma_{\mu} L_{i}\right)\left(\Phi^{\dagger} i D^{\mu} \Phi\right)+c_{3, i}\left(\bar{L}_{i} \gamma^{\mu} \tau^{a} L_{i}\right)\left(\Phi^{\dagger} \overleftrightarrow{i \tau^{a} D_{\mu}} \Phi\right) \\
& +h^{i j} \Phi^{\dagger} \Phi \bar{L}_{i} \Phi E_{j}+\text { h.c. },
\end{aligned}
$$

where $\overleftrightarrow{i \tau^{a} D_{\mu}}=1 / 2\left(i \tau^{a} D_{\mu}-i \overleftarrow{D}_{\mu} \tau^{a}\right) . \quad L_{i}\left(E_{i}\right)$ represents a lepton doublet (singlet) field of flavour $i$. The SM Higgs doublet is denoted by $\Phi$, and $B_{\mu \nu}$ and $W_{\mu \nu}^{A}$ are the field strength tensors of $\mathrm{U}(1)_{\mathrm{Y}}$ and $\mathrm{SU}(2)_{\mathrm{L}}$ gauge field, respectively.

The last three lines of (3) contain operators that contribute to radiative penguin transitions at the one loop-level in the effective theory, but can be generated at tree level in RS model. The operators in the first line correspond to dipole operators after electroweak symmetry breaking and therefore contribute at tree level. However, they are generated only by loops in the 5D theory. Once the corresponding matching coefficients have been computed in the RS model at tree- and one-loop level, respectively, the amplitude for the radiative dipole transition is obtained by the following steps. First, we parametrize the fields by the mass eigenstates in the broken theory below the electroweak scale, making the replacement

$$
\Phi \rightarrow \frac{1}{\sqrt{2}}\left(\begin{array}{c}
\sqrt{2} \phi^{+} \\
v+H+i G
\end{array}\right) \quad E_{i} \rightarrow V_{i j} P_{R} \psi_{j}, \quad L_{i} \rightarrow U_{i j} P_{L}\left(\begin{array}{c}
\nu_{j} \\
\psi_{j}
\end{array}\right)
$$

where $\psi_{i}$ is the Dirac spinor field for the massive leptons $(i=1,2,3$ corresponding to electron, muon, tau $)$ and $\nu_{i}$ is the corresponding neutrino spinor field. $P_{L / R}=\left(1 \mp \gamma_{5}\right) / 2$ are the chiral projectors. The unitary matrices $U$ and $V$ relate the interaction and mass flavour bases. Then we compute the dipole transition with the $4 \mathrm{D}$ effective theory. This requires the computation of the $\left(p+p^{\prime}\right)^{\mu}$ structure (related to $\sigma^{\mu \nu} q_{\nu}$ via the Gordon identity) of the diagrams shown in Fig. 1. The couplings in the mass basis are now given by

$$
\begin{aligned}
\alpha_{i j} & =\left[U^{\dagger} a V\right]_{i j}, \\
\beta_{i j k l} & =\sum_{m, n}\left[U^{\dagger}\right]_{i m} U_{m j}\left[V^{\dagger}\right]_{k n} V_{n l} b_{m n}, \\
\gamma_{1, i j} & =\sum_{m}\left[V^{\dagger}\right]_{i m} V_{m j} c_{1, m}, \\
\gamma_{x, i j} & =\sum_{m}\left[U^{\dagger}\right]_{i m} U_{m j} c_{x, m} \quad(x=2,3),
\end{aligned}
$$

with $a_{i j}=c_{W} a_{B, i j}-s_{W} a_{W, i j}$.

The calculation is straightforward and can be carried out with standard techniques. Let us mention a few subtleties:

- Though finite the diagrams must be evaluated in some regularization scheme. In dimensional regularization terms of the form $\epsilon \times \frac{1}{\epsilon}$ arise that would be missed in a purely four-dimensional calculation. 


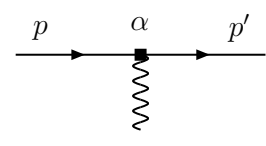

(a)

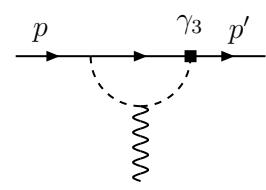

(d1)

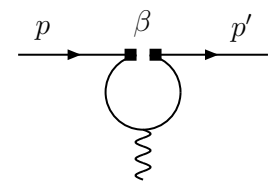

(b)

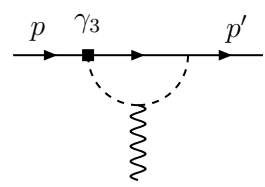

$(\mathrm{d} 2)$

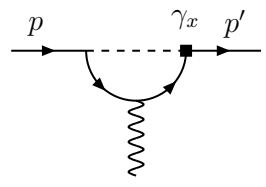

(c1)

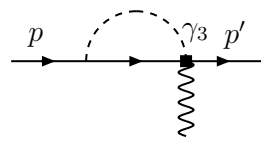

$(\mathrm{e} 1)$

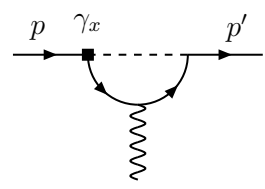

(c2)

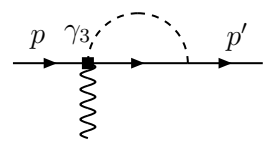

(e2)

Figure 1: Diagrams that contribute to $a_{\mu}$ in the $4 \mathrm{D}$ effective theory.

- The sum of all the one-loop diagrams in the effective theory is scheme dependent. This dependence cancels with the scheme dependence of the matching coefficient $\alpha_{i j}$ of the dipole operators that arises in the 5D loop calculation.

- In naive dimensional regularization (NDR, anti-commuting $\gamma_{5}$ ) only the dipole and fourlepton operators give a non-vanishing contribution (diagrams (a), (b) in Fig. 1).

Focusing on the muon anomalous magnetic moment, the result in NDR scheme is given by ${ }^{\mathrm{b}}$

$$
\Delta a_{\mu}=\frac{g_{\mu}-2}{2}=-\frac{4 m_{\mu}^{2}}{T^{2}}\left(\frac{\operatorname{Re}\left(\alpha_{22}\right)}{y_{\mu} e}+\sum_{k=1,2,3} \frac{1}{16 \pi^{2}} \frac{m_{\ell_{k}}}{m_{\mu}} \operatorname{Re}\left(\beta_{2 k k 2}\right)\right),
$$

where $\Delta a_{\mu}$ refers to the additional contributions generated by the KK excitations. Other observables such as the $\mu \rightarrow e \gamma$ or $\tau \rightarrow e \gamma$ branching fractions can be determined in an analogous fashion. The electric dipole moments are related to the imaginary parts of the matching coefficients. Note that we extracted the square of the muon mass by dividing by the small 4D muon Yukawa coupling $y_{\mu}$. This anticipates that to a very good approximation we shall find that the gauge-boson exchange contribution to $\alpha_{22}$ is proportional to $y_{\mu}$. The natural size of $\Delta a_{\mu}$ in the RS model is therefore of order $\alpha_{\mathrm{em}} /(4 \pi) \times m_{\mu}^{2} / T^{2} \approx 0.7 \cdot 10^{-11} \times(1 \mathrm{TeV} / T)^{2}$, far too small (unless $T$ is unrealistically small) to explain the present discrepancy between measurement and theoretical prediction, $a_{\mu}^{\exp }-a_{\mu}^{\mathrm{SM}}=239(63)(48) \times 10^{-11},{ }^{22}$ unless there is some additional parametric or numerical enhancement.

\section{5D Feynman rules}

The next and most important step is the determination of the Wilson coefficients in (3) from the underlying RS model. We perform the matching calculation in a manifestly 5D formalism rather than work with an infinite tower of KK modes. This avoids the calculation of KK sums but requires the knowledge of the $5 \mathrm{D}$ Feynman rules.

\footnotetext{
b The formula omits a small contribution of approximately $0.4 \cdot 10^{-11} \times(1 \mathrm{TeV} / T)^{2}$ from the $\gamma_{x, 22}$ couplings that can be interpreted as modifications of the gauge-boson-fermion vertices in the SM diagrams.
} 
The general strategy for their derivation was established in Refs. ${ }^{9,23}$ We refer the reader to the appendix of Ref. ${ }^{10}$ for a comprehensive summary. Since the RS model has ordinary translation invariance on four-dimensional hypersurfaces orthogonal to the fifth dimension, it is convenient to use a mixed momentum-coordinate space representation with four-dimensional, continuous momentum and a bulk position variable. The necessary ingredients for the calculation are then: 5D vertex rules, 5D propagators and the zero-mode wave functions. Since we integrate out scales far above the electroweak scale and match onto a set of $\mathrm{SU}(3) \times \mathrm{SU}(2) \times \mathrm{U}(1)$ invariant operators, it suggests itself to work in the $\mathrm{SU}(2) \times \mathrm{U}(1)$-symmetric phase. The wrongsign Higgs mass term is then a perturbation, and the zero-mode fermions and gauge bosons do not receive masses due to the Higgs mechanism. Their bulk wave functions are simply given by $^{24,25}$

$$
f_{\gamma}^{(0)}(z)=\sqrt{\frac{k}{\ln \frac{k}{T}}}, \quad \quad f_{L}^{(0)}(z)=\sqrt{\frac{1-2 c_{L}}{1-\left(\frac{T}{k}\right)^{1-2 c_{L}}}} \sqrt{T}(k z)^{2}(T z)^{-c_{L}}
$$

and a similar expression with $c_{L_{i}} \rightarrow-c_{E_{i}}$ for the right-handed zero-mode $g_{E_{i}}(z)$ of the singlet lepton field $E_{i}{ }^{\mathrm{c}}$. Note that reference to the KK zero modes cannot be avoided, since they correspond to the SM fields, which are not integrated out (unless highly virtual). Their wave functions appear on the external legs when the 5D Green functions are matched to those of the $4 \mathrm{D}$ effective theory.

Determining the Feynman rules is in principle straightforward. While the vertices are simple, the propagators require more work. As usual, they are found by inverting the differential operator in the bilinear terms of the action. For fermions, in the mixed representation, this requires solving

$$
\left[\frac{1}{k z}\right]^{4} \mathcal{D} \Delta\left(p, z, z^{\prime}\right)=i \delta\left(z-z^{\prime}\right) \mathbb{1} \quad \text { with } \quad \mathcal{D}=\not p+i \Gamma^{5}\left(\partial_{z}-\frac{2}{z}\right)-\frac{c}{z} .
$$

Since the 5D fermions are non-chiral, the propagator contains four chiral components,

$$
\begin{aligned}
\Delta_{L}(p, x, y)= & -\underbrace{P_{L} F_{L}^{+}(p, x, y) \not p P_{R}}_{\text {contains zero mode }}-P_{R} F_{L}^{-}(p, x, y) \not p P_{L} \\
& +\underbrace{P_{L} d^{+} F_{L}^{-}(p, x, y) P_{L}+P_{R} d^{-} F_{L}^{+}(p, x, y) P_{R}}_{\text {mass terms }}
\end{aligned}
$$

where

$$
\left[-p^{2}-\partial_{z}^{2}+\frac{c^{2} \pm c-6}{z^{2}}+\frac{4}{z} \partial_{z}\right] F^{ \pm}\left(p, z, z^{\prime}\right)=i(k z)^{4} \delta\left(z-z^{\prime}\right)
$$

and $d^{ \pm} \equiv \pm\left[\partial_{z}-(2 \pm c) / z\right]$. The interpretation is clear when written in terms of the KK decomposition, which reads, for example:

$$
F_{L}^{+}(p, x, y)=\sum_{n} f_{L}^{(n)}(x) \frac{-i}{p^{2}-m_{n}^{2}} f_{L}^{(n)}(y)
$$

\footnotetext{
${ }^{\mathrm{c}} c_{\psi}=M_{\psi} / k$ denotes the dimensionless parameter related to the Planck-scale 5D bulk mass $M_{\psi}$ of fermion field $\psi$. In general, for fermions, $f(g)$ denotes left-handed (right-handed) mode functions from the 4D perspective.
} 

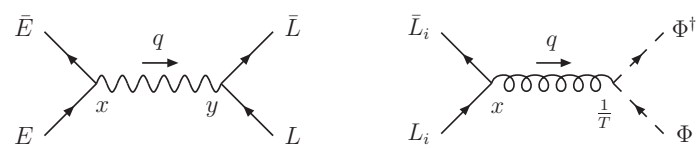

Figure 2: Left: Hypercharge boson exchange that generates the four-fermion operator $\left(\bar{L}_{i} \gamma^{\mu} L_{i}\right)\left(\bar{E}_{j} \gamma_{\mu} E_{j}\right)$. Right: SU(2) gauge boson exchange that generates the fermion-Higgs operators in (3).

$$
d^{-} F_{L}^{+}(p, x, y)=\sum_{n} g_{L}^{(n)}(x) \frac{i m_{n}}{p^{2}-m_{n}^{2}} f_{L}^{(n)}(y)
$$

In the 5D formalism, we solve (10) in terms of Bessel functions. Introducing

$$
\begin{aligned}
& S_{ \pm}(p, x, y, c)=I_{c \pm 1 / 2}(p x) K_{c \pm 1 / 2}(p y)-K_{c \pm 1 / 2}(p x) I_{c \pm 1 / 2}(p y) \\
& \tilde{S}_{ \pm}(p, x, y, c)=I_{c \pm 1 / 2}(p x) K_{c \mp 1 / 2}(p y)+K_{c \pm 1 / 2}(p x) I_{c \mp 1 / 2}(p y),
\end{aligned}
$$

the Euclidean propagator is given by expressions such as

$$
\begin{aligned}
d^{+} F_{L}^{-}(p, x, y)= & -p \Theta(x-y) \frac{i k^{4} x^{5 / 2} y^{5 / 2} \tilde{S}_{+}\left(p, x, 1 / T, c_{L}\right) S_{-}\left(p, y, 1 / k, c_{L}\right)}{S_{-}\left(p, 1 / T, 1 / k, c_{L}\right)} \\
& -p \Theta(y-x) \frac{i k^{4} x^{5 / 2} y^{5 / 2} S_{-}\left(p, y, 1 / T, c_{L}\right) \tilde{S}_{+}\left(p, x, 1 / k, c_{L}\right)}{S_{-}\left(p, 1 / T, 1 / k, c_{L}\right)} .
\end{aligned}
$$

We note (for later) that the propagator is discontinuous at $x=y$. Similar expressions are found for the other propagator components and for $\mathrm{SU}(2)$ singlet fermion and gauge fields.

\section{Matching: Tree-level operators}

With the 5D propagators at hand the determination of the tree-level matching coefficients of the four-lepton operator $\left(\bar{L}_{i} \gamma^{\mu} L_{i}\right)\left(\bar{E}_{j} \gamma_{\mu} E_{j}\right)$ and the lepton-Higgs operators in (3) becomes trivial. The relevant diagrams are shown in Fig. 2.

Since the lepton-Higgs operators do not contribute to the anomalous magnetic moment in the NDR scheme, see (6), we only discuss the four-lepton operator. It can be generated only by hypercharge gauge boson exchange. The diagram readily translates into an expression for the Wilson coefficient:

$$
b_{i j}=-i\left(i g_{5}^{\prime}\right)^{2} \frac{Y_{L}}{2} \frac{Y_{E}}{2} T^{2} \int_{1 / k}^{1 / T} d x d y \frac{f_{L_{i}}^{(0)^{2}}(x)}{(k x)^{4}} \frac{g_{E_{j}}^{(0)^{2}}(y)}{(k y)^{4}} \Delta_{\perp}^{\mathrm{ZMS}}(q=0, x, y) .
$$

$\Delta_{\perp}^{\mathrm{ZMS}}$ refers to the $5 \mathrm{D}$ propagator with the massless zero-mode subtracted, since zero-mode exchange is a low-energy effect and not part of the matching coefficient. Once the zero mode is subtracted, the external momenta can be set to zero, implying $q=0$. In this limit, the gauge-boson propagator $\Delta_{\perp}(q, x, y)$ has a particularly simple structure:

$$
\Delta_{\perp}(q, x, y) \stackrel{q \rightarrow 0}{=} \Theta(x-y) \frac{i k}{\ln \frac{k}{T}}\left(-\frac{1}{q^{2}}+\frac{1}{4}\left\{\frac{1 / T^{2}-1 / k^{2}}{\ln \frac{k}{T}}-x^{2}-y^{2}+2 x^{2} \ln (x T)\right.\right.
$$




$$
\left.\left.+2 y^{2} \ln (y T)+2 y^{2} \ln \frac{k}{T}\right\}+\mathcal{O}\left(q^{2}\right)\right)+(x \leftrightarrow y)
$$

The singular piece $\propto 1 / q^{2}$ arises from the massless zero mode that needs to be subtracted. After inserting (15) into (14) all integrals are elementary. The result agrees with the calculation of four-quark operators in the KK mode language. ${ }^{21}$

Inserting the Wilson coefficient into (6) gives a contribution to the anomalous magnetic moment of

$$
\Delta a_{\mu}=\frac{\alpha_{\mathrm{em}}}{8 \pi c_{W}^{2}} \frac{m_{\mu}^{2}}{T^{2}} \frac{1}{\ln \frac{k}{T}} f\left(\ln (k / T), c_{L}, c_{E}\right) \approx 1.2 \times 10^{-13} \times \frac{(1 \mathrm{TeV})^{2}}{T^{2}} .
$$

The function $f$ is close to one, resulting in the numerical estimate above. This is far below the current experimental and theoretical uncertainties for any allowed value of the KK scale $T$, since it is suppressed relative to the naive estimate by the large factor $\ln \frac{k}{T} \approx 35$.

\section{5D penguin diagrams - gauge boson exchange}

The RS model does not generate the dimension-six dipole operators at tree-level. The matching calculation for the dipole operators therefore requires the calculation of genuine 5D one-loop diagrams. There are two classes of diagrams that enter the matching procedure: diagrams with an internal gauge-boson propagator, shown in Fig. 3, and diagrams with internal Higgs exchange. We first discuss the gauge-boson contributions, which are technically more difficult but conceptually simpler than the Higgs diagrams, discussed in the following section.

\subsection{The calculation}

The calculation of the one-loop coefficients $a_{i j}$ can be simplified by restriction to an external photon, i.e. the linear combination $c_{W} B_{\mu}+s_{W} W_{\mu}^{3}$, which reduces the number of diagrams (slightly). In addition, we ignore from the start terms that vanish when the Higgs doublet in the operators $\bar{L}_{i} \Phi \sigma_{\mu \nu} E_{j} B^{\mu \nu}, \bar{L}_{i} \tau^{a} \Phi \sigma_{\mu \nu} E_{j} W^{a, \mu \nu}$ is set to its vacuum expectation value. All non-vanishing one-loop diagrams are shown in Fig. 3.

The matching coefficients must only absorb quantum effects related to the short distance scales $T$ and $k$. In general, however, a one-loop diagram has three distinct parts:

(1) A part where each propagator only propagates the zero mode. This is obviously part of the SM contribution to the penguin amplitude and must be removed. It turns out that this can be achieved by subtracting the zero mode from only the gauge-boson propagators. The reason is that the presence of a gauge-boson zero mode automatically forces all other propagators to only propagate a zero-mode due to orthogonality relations and the fact that all external states are zero modes.

(2) At least one propagator contains a KK mode, but the $4 \mathrm{D}$ loop momentum $l$ is much smaller than the the scale $T$. The subgraph consisting of KK mode propagators can be contracted to point, which corresponds to the insertion of a higher-dimensional operator into a $4 \mathrm{D}$ graph. In most cases (as when more that one KK propagator is present) the corresponding operator is of a dimension higher than six and can be ignored. The remaining dimension-six operator insertions precisely correspond to the one-loop matrix 

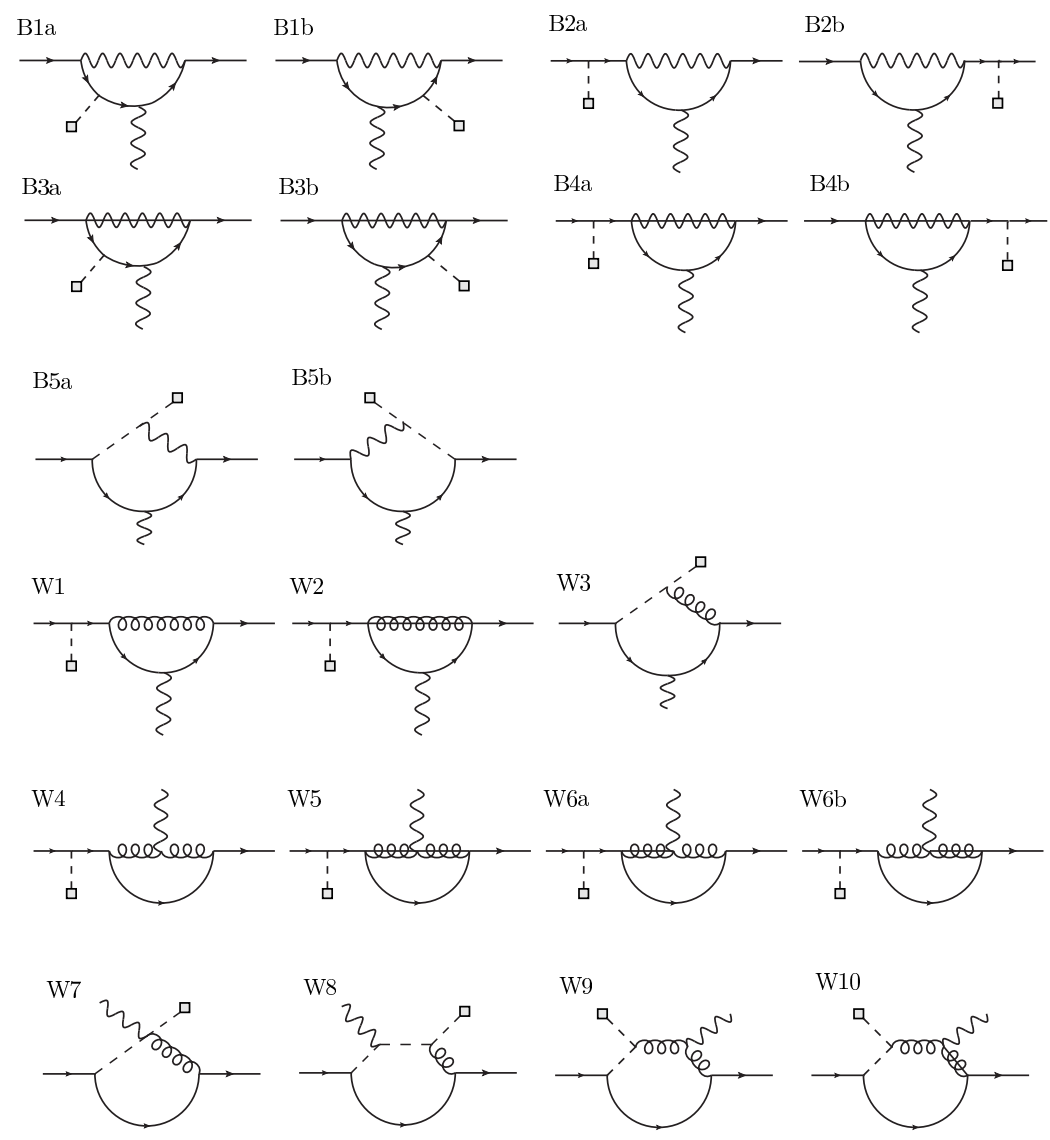

Figure 3: Diagrams contributing to the matching coefficients of the dimension-six dipole operators. Solid lines refer to leptons, with the right external line belonging to the doublet $L_{i}$, the left one to $E_{j}$. Wavy lines denote hypercharge gauge bosons and the external photon, curly lines $\mathrm{SU}(2) \mathrm{W}$-bosons. A solid-wavy (solid-curly) line refers to the scalar fifth component of the gauge field. Dashed lines denote Higgs bosons, including the external Higgs field (grey box). Vertices involving Higgs fields are localized at $1 / T$, all other vertices are integrated over position in the fifth dimension.

elements of the non-dipole operators in (3) with tree-level matching coefficients as determined in the previous section. An example is diagram B1a/b in Fig. 3. The gauge-boson propagator must propagate KK modes (see above). If the fermion lines are zero modes then the short-distance subgraph is the four-lepton operator, and the contracted diagram corresponds to the insertion of this operator as in diagram (b) of Fig. 1.

(3) Finally, we have the contribution where the loop momentum is of the order $l \sim T$ or larger. Only this is part of the Wilson coefficient $a_{i j}$. This contribution can be extracted directly by expanding the integrand in the lepton external momenta $p$ and $p^{\prime}$ (after subtracting the zero mode from the gauge-boson propagator). The expansion is usually only necessary to the first non-trivial order as a dipole operator $\bar{L} \Phi \sigma^{\mu \nu} E F_{\mu \nu}$ is linear in the external momenta (see, however, below).

The remaining calculation is tedious and requires a combination of further analytical sim- 
plifications and final numerical integrations. To give an example, we consider again diagram B1. We decompose each fermion propagator into its four chiral components using (9). Most of the 64 possible terms vanish due to the chiral projectors $P_{R}$ and the brane boundary conditions $g_{L_{i}}(1 / T)=f_{E_{j}}(1 / T)=0$. The two remaining terms result in

$$
\begin{aligned}
\mathbf{B 1 a}= & \frac{g_{5}^{\prime 2} e_{5} Q_{\mu} Y_{L} Y_{E} y_{i j}^{(5 \mathrm{D})} T^{3}}{4 k^{3}} \int_{1 / k}^{1 / T} \frac{d x}{(k x)^{4}} \int_{1 / k}^{1 / T} \frac{d y}{(k y)^{4}} \int_{1 / k}^{1 / T} \frac{d z}{(k z)^{4}} \int \frac{d^{4} l}{(2 \pi)^{4}} \\
& f_{L_{i}}^{(0)}(z) f_{\gamma}^{(0)}(y) g_{E_{j}}^{(0)}(x) \epsilon^{* \mu} \Delta_{\mathrm{ZMS}}^{\rho \nu}(l, x, z) \\
& \bar{L}_{i}\left(p^{\prime}\right)\left[F_{L_{i}}^{+}\left(\hat{p}^{\prime}, z, y\right) F_{L_{i}}^{+}(\hat{p}, y, 1 / T) F_{E_{j}}^{-}(\hat{p}, 1 / T, x)\left\{\gamma_{\rho}\left(p^{\prime} \not t\right) \gamma_{\mu} \gamma_{\nu}\right\}(p-l)^{2}+\right. \\
& \left.d^{+} F_{L_{i}}^{-}\left(\hat{p}^{\prime}, z, y\right) d^{-} F_{L_{i}}^{+}(\hat{p}, y, 1 / T) F_{E_{j}}^{-}(\hat{p}, 1 / T, x)\left\{\gamma_{\rho} \gamma_{\mu}(p-\not) \gamma_{\nu}\right\}\right] P_{R} E_{j}(p),
\end{aligned}
$$

where $\hat{p}=p-l, \hat{p}^{\prime}=p^{\prime}-l$. This provides the starting point for the above-mentioned expansion in $p$ and $p^{\prime}$. The numerical integrations include the modulus of the 4D Euclidean loop momentum $l$ and the three bulk coordinates. Considerable numerical speed-up and improved accuracy can be achieved by carrying out the integration of the photon vertex bulk position $y$ analytically. We refer to Ref. ${ }^{10}$ for the details and add here only a few comments.

Scheme (in)dependence - The scheme dependence of the one-loop diagrams (b) to (e) in Fig. 1 must be cancelled by the scheme dependence of the one-loop dipole coefficient $a_{i j}$. The scheme-dependence of the short-distance contributions arises from diagrams B1a/b and W8 that are potentially IR singular after the expansion in the external momenta $p$ and $p^{\prime}$. As was the case for diagrams (b) to (e), there is in fact no singularity due to evanescent numerators. However, a naive treatment in $d=4$ dimension misses finite terms of the form $\varepsilon_{I R} \times \frac{1}{\varepsilon_{I R}}$. These terms have to be added "by hand" by computing analytically the difference between the correct $d$-dimensional and naive four-dimensional treatment. They precisely cancel the scheme dependence due to the tree-level operator insertions. After this we can safely work in $d=4$ dimensions and use numerical methods to determine the short-distance coefficients.

One-particle reducible (1PR), "off-shell" contributions - 1PR diagrams such as B2a contribute to the short-distance coefficient. The fermion propagator that connects the external Higgs insertion to the loop is

$$
\Delta_{i}^{L}(p, x, 1 / T) P_{R}=-F_{L_{i}}^{+}(p, x, 1 / T) \not p P_{R}+d^{-} F_{L_{i}}^{+}(p, x, 1 / T) P_{R} .
$$

The second term on the right-hand side propagates only KK excitations and is purely shortdistance. The first term vanishes by the on-shell condition $\not p u(p, s)=0$ except for the zero-mode contribution

$$
\Delta_{\mathrm{ZM}}(p, x, 1 / T)=f_{L_{i}}^{(0)}(x) \frac{i \not p}{p^{2}} P_{R} f_{L_{i}}^{(0)}(1 / T)
$$

If the one-particle pole at $p^{2}=0$ remains in the final answer, this is a clear sign for a longdistance effect and this part of the external Higgs insertion into a zero mode needs to be amputated. (In fact there is an infinite series of external Higgs insertions connected by zeromode propagators that necessarily sums to the massive SM lepton propagator.) However, when the $1 / p^{2}$ factor is cancelled by numerators, we obtain an additional contribution to the 

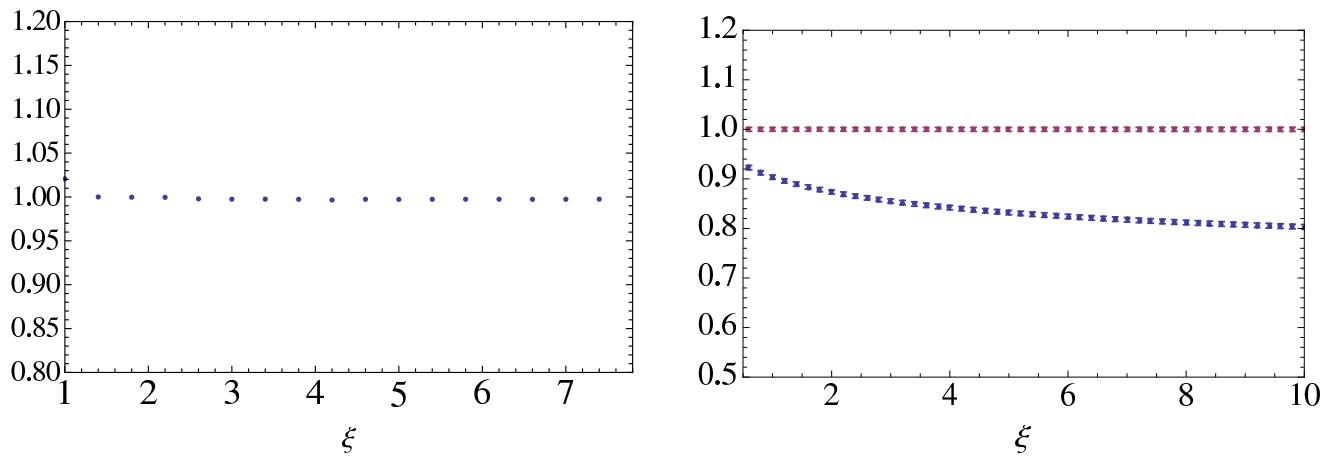

Figure 4: Left panel: Residual gauge-parameter dependence of the short-distance coefficient $a_{22}$ in the minimal RS model normalized to its value for $\xi=1$ for $c_{L_{2}}=-c_{E_{2}}=0.5748$. Right panel: As left panel but for the gauge-invariant subset of all $Z_{X}$-diagrams in the custodially protected RS model for $c_{L_{i}}=0.1$ and $c_{E_{j}}=-1.2$. The lower curve is without off-shell terms, the upper line includes the off-shell terms.

matching coefficient. If we parametrize the one-particle irreducible $\bar{L}_{i} L_{i} \gamma$ vertex subdiagram with off-shell zero-mode fermions as

$$
\Lambda^{\mu}=\Lambda_{\mathrm{on}}^{\mu}+\not p^{\prime} \Lambda_{\text {off, } \mathrm{p}^{\prime}}^{\mu}+\Lambda_{\text {off, } \mathrm{p}}^{\mu} \not p,
$$

the piece of interest arises from the $\Lambda_{\text {off, } \mathrm{p}}^{\mu} \not p$ term, and is given by

$$
\Lambda^{\mu} \Delta_{\mathrm{ZM}}(p, x, 1 / T)=i \Lambda_{\text {off }, \mathrm{p}}^{\mu} f_{L_{i}}^{(0)}(x) f_{L_{i}}^{(0)}(1 / T) .
$$

Note that to compute this piece for the dipole transition, we need to expand the diagram to second order in the external momenta to extract the coefficient of the $p^{\mu}$ and $p^{\prime \mu}$ terms. For the standard choices of the bulk mass parameters of the left- and right-handed fermions, we find that these "off-shell" terms are numerically suppressed.

Gauge invariance - We performed the computation in general covariant 5D gauge and verified the gauge-parameter independence analytically by using algebraic equation-of-motion and integration-by-parts identities. A sketch of the proof is given in Ref. ${ }^{10}$ The $1 \mathrm{PR}$ contributions are required to make the result gauge invariant. We also perform the numerical calculation at different gauge parameters and use the residual dependence as a check and diagnostic for the numerical uncertainty. In this way we verify gauge-parameter independence numerically with $0.5 \%$ accuracy. The left panel of Fig. 4 shows the residual gauge-parameter dependence of the short-distance coefficient $a_{22}$ for the symmetric bulk mass parameters $c_{L_{2}}=-c_{E_{2}}=0.5748$. For usual choices for the 5D mass parameters the off-shell terms are negligibly small and below the numerical accuracy. However, if one of the fermion zero modes is IR localized (as would be the case for the right-handed top) their effect becomes visible. To illustrate that the off-shell terms are required for gauge independence we show in the right panel of Fig. 4 the gauge-invariant subset of diagrams with a $Z_{X}$ boson that contributes to $a_{i j}$ in the custodially protected RS model (see Section 8). ${ }^{\mathrm{d}}$ Here $c_{L_{i}}=0.1$ and $c_{E_{j}}=-1.2$ are chosen. The plot shows the gauge-parameter dependence of the contribution to $a_{i j}$ with (upper points) and without (lower points) off shell terms normalized to the value for $\xi=1$.

\footnotetext{
${ }^{\mathrm{d}}$ For diagrams with internal $Z_{X}$ bosons the numerical accuracy is highest as we do not need to subtract zero modes.
} 


\subsection{Numerical result}

The gauge-boson exchange contribution to $g_{\mu}-2$ is almost insensitive to the structure of the $5 \mathrm{D}$ Yukawa matrices and bulk mass parameters and can be expressed in terms of known lowenergy parameters and the scales $T$ and $k$. To understand this point it is convenient to write the gauge-boson contribution $\Delta a_{i j}^{\mathrm{g}}$ to the short-distance coefficient in the form

$$
\Delta a_{i j}^{\mathrm{g}}=y_{i j}^{(5 D)} \frac{T^{3}}{k^{4}} f_{L_{i}}^{(0)}(1 / T) g_{E_{j}}^{(0)}(1 / T) \mathcal{A}_{i j},
$$

extracting the Yukawa matrix from the single Higgs insertion in Fig. 3 and the external fermion zero-mode wave-functions. We then find numerically that $\mathcal{A}_{i j} \approx \mathcal{A}$ varies only mildly with the $5 \mathrm{D}$ mass parameters $c_{i}$, whereas $\Delta a_{i j}^{\mathrm{g}}$ itself is strongly model parameter dependent. However, when $\mathcal{A}_{i j}$ does not depend on $i j$, the rotation to the lepton-mass eigenbasis simply turns the extracted terms into the diagonal lepton-mass matrix, hence to very good approximation $\Delta a_{\mu}^{\mathrm{g}}=\sqrt{2} m_{\mu} / v \times \mathcal{A}$, independent of $y_{i j}^{(5 D)}$ and bulk masses. For the same reason, lepton-flavour violating effects from gauge-boson exchange diagrams are strongly suppressed.

Thus, the gauge contribution to $g_{\mu}-2$ can the be approximated with accuracy of a few percent by

$$
\Delta a_{\mu}^{\mathrm{g}} \approx 0.25 \cdot 10^{-11} \times \log \frac{k}{T} \times \frac{(1 \mathrm{TeV})^{2}}{T^{2}} \approx 8.8 \cdot 10^{-11} \times \frac{(1 \mathrm{TeV})^{2}}{T^{2}}
$$

The overall scaling factor with $T^{-2}$ is a general feature of dimension-six operator effects, while the additional logarithm arises from the zero-mode subtracted gauge-boson propagator. The remaining (implicit) $T$ and $k$ dependence is negligible. Note that compared to the four-lepton operator contribution (16) the present one is enhanced (rather than suppressed) by the large $\operatorname{logarithm} \log (k / T) \approx 35$. The enhancement arises from the propagation of the internal KK fermions. The numerical prefactor 0.25 is however smaller than the naive parametric estimate 0.7. Due to the approximate independence of the theoretical computation on the Yukawa structure and bulk-mass parameters, the muon anomalous magnetic moment provides a robust constraint on the KK scale of the RS model, similar to the electroweak precision $S$ - and $T$ parameters.

Overall, for a lowest $\mathrm{KK}$ excitation of mass $1 \mathrm{TeV}$, the gauge-boson contribution is of order of the present experimental and theoretical uncertainty in $g_{\mu}-2$. It remains, however, about a factor 5 below the the present difference between the central experimental and theoretical values, though the shift $\Delta a_{\mu}^{g}$ has the right sign to reduce it. Since lowest KK excitations with mass $1 \mathrm{TeV}$ are already excluded even by direct searches, the anomalous magnetic moment currently provides no competitive lower limit on $T$ in the minimal RS model.

\section{5D penguin diagrams - Higgs exchange}

The previous discussion ignored contributions from internal Higgs exchange diagrams. These diagrams are proportional to a different flavour structure, containing three Yukawa coupling factors. ${ }^{\mathrm{e}}$ There are only three non-vanishing one-loop diagrams in the minimal RS model, shown in Fig. 5. Note that the external Higgs field necessarily couples to an external fermion

\footnotetext{
${ }^{\mathrm{e}}$ An odd number of Yukawa couplings is required to convert an external SU(2) doublet zero mode into an
} external $\mathrm{SU}(2)$ singlet one. 

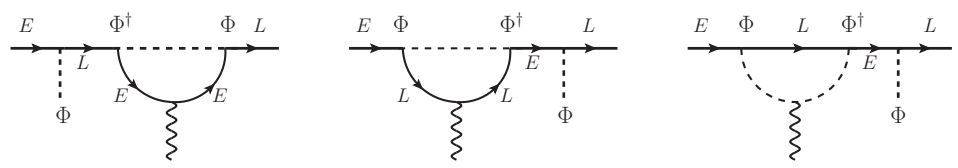

Figure 5: Higgs-boson exchange diagrams. Non-vanishing contributions from these diagrams either require a wrong-chirality Higgs coupling or the cancellation of the external propagator.

line. The coupling to a fermion inside the loop leads to dimension-eight operators such as $\bar{L}_{i} \Phi \sigma^{\mu \nu} E_{j} F_{\mu \nu} \Phi^{\dagger} \Phi$.

Each diagram in Fig. 5 provides two distinct contributions to the dipole-operator matching coefficient. The first arises from the off-shell vertex function that was discussed already in relation with the gauge-boson diagrams. Its computation is straightforward and can be carried out analytically, since due to the brane localization of the Higgs field there is only one bulkcoordinate integration from the photon vertex. The resulting contribution is quite small. ${ }^{\mathrm{f}}$ The second contribution arises from what has been discussed in the literature ${ }^{5,26,27}$ under the name of "wrong chirality Higgs couplings" (WCHCs). In the context of radiative transitions, previous references to WCHC in the KK picture appear in Refs., ${ }^{11,12}$ where the effect of the lowest quark KK mode is studied. Consider the coupling of the brane-localized Higgs field to the $\mathrm{SU}(2)$ singlet and doublet leptons,

$$
\int d^{4} x[(\bar{L} \Phi) E+\text { h.c. }]_{z=1 / T}=\int d^{4} x\left[\left(\bar{L}_{L} \Phi\right) E_{R}+\left(\bar{L}_{R} \Phi\right) E_{L}+\text { h.c. }\right]_{z=1 / T},
$$

where the 5D fields are split into their 4D chirality components. The second, "wrong-chirality Higgs coupling" term on the right-hand side is obviously absent in the SM, but a priori present in the RS model, since the KK excitations are non-chiral. However, the boundary conditions of the right-handed $\mathrm{SU}(2)$ doublet and left-handed $\mathrm{SU}(2)$ singlet require the wrong-chirality fields to vanish on the IR brane, so the WCHCs vanish for a brane-localized Higgs. This expectation turns out to be too naive, since an exactly, delta-function localized Higgs cannot be unambiguously defined. ${ }^{26}$ In the 5D formalism applied to the unbroken electroweak theory, an exactly localized Higgs would require the evaluation of the discontinuous 5D propagators at the location of the discontinuity. To avoid this ambiguity we define the RS model with brane-localized Higgs through the limit of a model with a Higgs profile with a small width $\delta / T$, where $\delta \ll 1$. A possible choice for such a regularized profile is

$$
\Phi(x, z)=\Phi(x) \frac{T}{\delta} \Theta(z-(1-\delta) / T) .
$$

For any finite $\delta$ the WCHC are not zero. Moreover, the Higgs profile introduces the new scale $T / \delta$ into the problem. It turns out that after integration over all bulk coordinates a contribution from the WCHC may survive in the limit $\delta \rightarrow 0$ that arises from the loop momentum region $l \sim T / \delta$. The loop integrand is illustrated in left panel of Fig. 6, where the blue (dark) curve differs from the red (grey) curve by a factor 10 smaller value of $\delta$.

\footnotetext{
f The smallness comes from the external zero-mode propagator. For "moderate" choices of the 5D mass parameters this contribution is usually negligible. This is different if one or more of the fermion zero-mode profiles are localized towards the IR brane, see Section 5.1.
} 

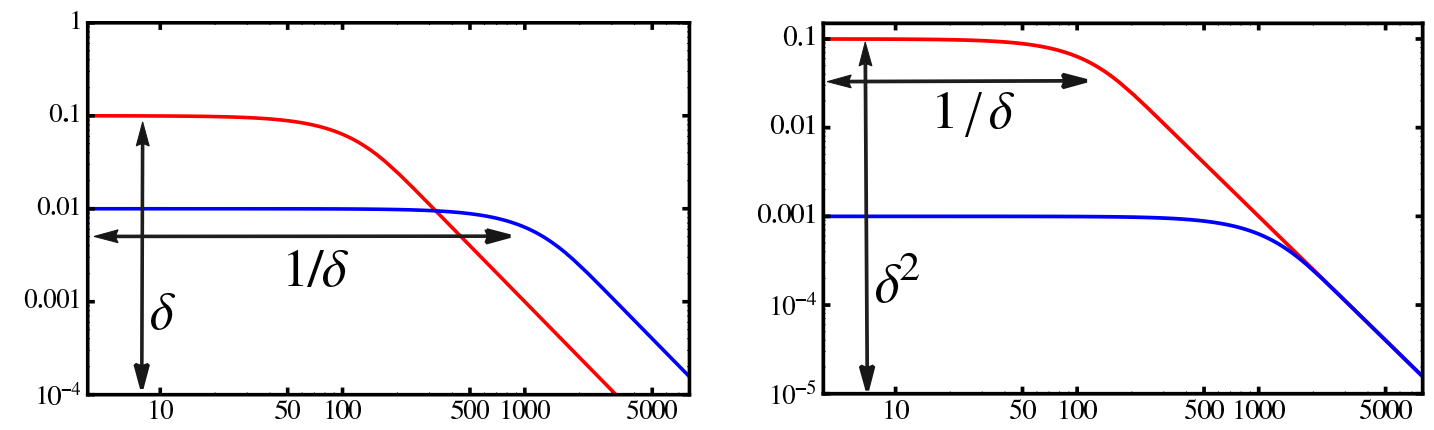

Figure 6: Qualitative behaviour of the loop momentum integrand (as function of $l$ in units of $T$ ) after integration over bulk coordinates and angles leaving only modulus $l$ for Higgs (left) and gauge-boson (right) exchange diagrams. The blue (dark) curve differs from the red (grey) curve by a factor 10 smaller value of $\delta$.

The result now depends on the precise meaning of "brane localization", when the RS model is itself interpreted as an effective field theory up to some scale $\Lambda$. "Exact brane localization" would imply that we take the limit $T / \delta \rightarrow \infty$ at fixed $\Lambda$. In this case, the WCHC contribution vanishes, since the area under the curve in the left panel of Fig. 6 up to $l=\Lambda$ is of order $\delta \times \Lambda$. However, the RS model with localized Higgs can also be interpreted as the idealization of a model with a Higgs field that lives parametrically near but not exactly on the IR brane. Then, if $T \ll T / \delta \ll \Lambda$, that is, when the limit $\Lambda \rightarrow \infty$ is taken before $T / \delta \rightarrow \infty$, we obtain a non-vanishing, model-independent WCHC contribution, since the length times height of the plateau in Fig. 6 approaches a finite limit. If, however, $T / \delta \sim \Lambda$, the result depends on the details of the Higgs profile. ${ }^{\mathrm{g}}$

Focusing on the two limiting cases, we find that for $\delta \rightarrow 0$ the contribution to the shortdistance coefficient is independent of the $5 \mathrm{D}$ mass parameters and obtain the compact expression

$$
\frac{1}{T^{2}} a_{i j}^{\mathrm{WCHC}}=\frac{e}{16 \pi^{2}} \frac{c y_{i j}^{\mathrm{SM}}}{T^{2}} \times \frac{\left[Y Y^{\dagger} Y\right]_{i j}}{Y_{i j}}
$$

with

$$
c= \begin{cases}-\frac{1}{12} & \Lambda \rightarrow \infty, \text { then } \delta \rightarrow 0 \\ 0 & \delta \rightarrow 0, \text { then } \Lambda \rightarrow \infty\end{cases}
$$

and $Y_{i j}=y_{i j}^{(5 \mathrm{D})} k$. Another WCHC Higgs contribution to the electromagnetic dipole transitions at order $1 / T^{2}$ comes from the operator $h_{i j} \bar{L}_{i} \Phi E_{j} \Phi^{\dagger} \Phi+$ h.c. in (3), since $h_{i j}$ is non-zero only when the wrong-chirality Higgs couplings are taken into account. The operator is generated at tree-level. With the step-function Higgs profile (25), the coefficient function reads (see also Ref. $^{27}$ )

$$
\frac{1}{T^{2}} h_{i j}=\frac{y_{i j}^{\mathrm{SM}}}{3 T^{2}} \times \frac{\left[Y Y^{\dagger} Y\right]_{i j}}{Y_{i j}} .
$$

\footnotetext{
${ }^{\mathrm{g}} \mathrm{A}$ similar non-commutativity of limits appears in the RS calculation of Higgs production, ${ }^{5}$ where it was discussed in the context of the KK-decomposed theory in the phase of broken electroweak theory.
} 
When two of the Higgs fields in $\bar{L}_{i} \Phi E_{j} \Phi^{\dagger} \Phi$ are put to their vacuum expectation values, this operator modifies the SM Yukawa couplings and leads to flavour-changing couplings of the zeromode fermions to the Higgs boson. Inserting this vertex into the Higgs-exchange contribution to the electromagnetic dipole transition similar to diagrams (c) and (d) of Fig. 1, we find that the result is suppressed relative to (26) by a factor of [lepton mass] ${ }^{2} / m_{H}^{2}$, where $m_{H}$ is the physical Higgs mass. The additional lepton-mass factors arise from the 4D Yukawa coupling at one of the Higgs-fermion vertices and the need for a helicity flip in the loop. Thus, the Higgs-exchange contribution to the anomalous magnetic moment and to radiative leptonflavour violating transitions from loop momentum $l \sim m_{H}$ is strongly suppressed relative to the contribution (26) that is generated at the KK scale. This effect is quite generic and implies that limits on radiative lepton-flavour violating decays usually impose much stronger constraints on the observability of lepton-flavour violating Higgs decays than assumed in Ref. ${ }^{28}$

Before turning back to numerical estimates, we briefly comment on potential WCHC contributions to the gauge-boson exchange diagrams. While present in principle, we find them vanishing at one-loop in the limit $\delta \rightarrow 0$ irrespective of the order of limits in $\delta$ and $\Lambda$. This can be understood when one keeps in mind that in the $5 \mathrm{D}$ formalism the WCHC emerge due to the discontinuity of the fermion propagator at coincident points. For a given loop momentum $l$ the leading contribution comes from region where the 5D coordinates $x, y$ in the fermion propagator $\Delta(l, x, y)$ are within a typical distance of $1 / l$. A fermion propagator that connects two Higgs vertices is essentially always near the coincident limit for $\delta \rightarrow 0$. For fermion propagators that connect a gauge-boson vertex with a Higgs vertex the coincidence requirement imposes an additional suppression factor, since the gauge boson is not localized near the brane. This is exemplified in the right panel of Fig. 6, where the behaviour of the WCHC terms of diagram $\mathrm{B} 1 \mathrm{a} / \mathrm{b}$ on $\delta$ is shown. The integral over the modulus of $l$ now vanishes as $\delta \rightarrow 0$, since the height of the plateau scales as $\delta^{2}$.

\section{Combined result}

Adding the Higgs-exchange contributions to the pure gauge-boson exchange result (23), we obtain

$$
\Delta a_{\mu} \approx\left[8.8+2.4\left\langle Y Y^{\dagger}\right\rangle_{\mu}\right] \cdot 10^{-11} \times \frac{(1 \mathrm{TeV})^{2}}{T^{2}},
$$

where the dimensionless quantity

$$
\left\langle Y Y^{\dagger}\right\rangle_{\mu}=\frac{\operatorname{Re}\left[\sum_{l m}\left[U^{\dagger}\right]_{2 l} f_{L_{l}}^{(0)}(1 / T)\left[Y Y^{\dagger} Y\right]_{l m} g_{E_{m}}^{(0)}(1 / T) V_{m 2}\right]}{\sum_{l m}\left[U^{\dagger}\right]_{2 l} f_{L_{l}}^{(0)}(1 / T) Y_{l m} g_{E_{m}}^{(0)}(1 / T) V_{m 2}}
$$

parametrises the flavour dependence of the contribution from (26) to $g_{\mu}-2$ compared to a term with only a single Yukawa matrix. ${ }^{\text {h }}$ Several comments should be made on this result.

(1) The Higgs-exchange contribution is strongly model-dependent in two ways: first, it depends on the entries of the unknown 5D Yukawa matrices. Second, it depends on the precise notion of Higgs localization. In particular, in the case of exact localization $(\delta \rightarrow 0$ first), the term proportional to $\left\langle Y Y^{\dagger}\right\rangle_{\mu}$ is absent altogether. The leading Higgs contribution then arises from dimension-eight operators and the "off-shell" terms. A subset of the former was calculated

\footnotetext{
${ }^{\mathrm{h}}$ The denominator of $(30)$ is proportional to the $4 \mathrm{D}$ muon Yukawa coupling $y_{\mu}$.
} 
for flavour-violating observables in Ref., ${ }^{9}$ where, on the other hand, the WCHC terms were not considered.

(2) In the opposite limit ( $\Lambda \rightarrow \infty$ first) the Higgs contribution as given above is the largest contribution to the anomalous magnetic moment, if the average Yukawa coupling is somewhat larger than one. With experimental and theoretical errors added linearly, the measurement of $g_{\mu}-2$ can then translated into the bound

$$
-25<\left\langle Y Y^{\dagger}\right\rangle_{\mu} \times(1 \mathrm{TeV} / T)^{2}<260
$$

where we required that $a_{\mu}^{\exp }-a_{\mu}^{\mathrm{RS}}$ stays compatible with zero at the $3 \sigma$ level.

(3) When we assume that all entries of the 5D Yukawa matrix are of the same order with no cancellations ("anarchic structure"), we can constrain the Higgs contribution to $g_{\mu}-2$ to be negligible relative to the gauge-boson one in a model-independent way from the non-observation of the $\mu \rightarrow e \gamma$ decay. Since the gauge contribution to $a_{i j}$ is almost flavour-aligned with the mass matrix its effect on FCNCs is suppressed and Higgs exchange is the dominant source of the $\mu \rightarrow e \gamma$ transition. Assuming

$$
\frac{\left[Y Y^{\dagger} Y\right]_{i j}}{Y_{i j}} \equiv Y_{\star}^{2}
$$

independent of $i j$ ("anarchy"), we find for the branching ratio

$$
\operatorname{Br}(\mu \rightarrow e \gamma)=6|c|^{2} \frac{\alpha_{\mathrm{em}}}{4 \pi} \frac{m_{e}}{m_{\mu}} \frac{Y_{\star}^{4}}{G_{F}^{2} T^{4}},
$$

where $c=-\frac{1}{12}$ as long as $\Lambda \gtrsim T / \delta$. Using the current MEG bound ${ }^{29} \operatorname{Br}(\mu \rightarrow e \gamma)<5.7 \cdot 10^{-13}$ we obtain

$$
Y_{\star} \times \frac{1 \mathrm{TeV}}{T}<0.16
$$

This by itself provides a strong constraint on the size of the Yukawa couplings (in combination with the RS scale $T$ ), which corresponds to Kaluza-Klein masses above $15 \mathrm{TeV}$ for $Y_{\star}=1$.

(4) Hence, under the assumption of Yukawa anarchy, we obtain the relation

$$
\Delta a_{\mu}^{\mathrm{WCHC}}=\sqrt{\frac{m_{\mu}}{m_{e}}} \frac{G_{F} m_{\mu}^{2}}{\pi \sqrt{6} e} \times \sqrt{\mathrm{Br}(\mu \rightarrow e \gamma)} \leq 0.6 \cdot 10^{-12},
$$

independent of the relation of $\delta$ and $\Lambda$ as well as the KK scale $T$. In this case the Yukawa sector and bulk-mass independent gauge-boson contribution is by far dominant.

\section{RS model with custodial protection}

The minimal RS model is severely constrained by the $T$-parameter, which is generated at treelevel with a $\log \frac{k}{T}$ enhancement. ${ }^{20} \mathrm{RS}$ models with custodial protection reduce this electroweak precision constraint allowing a lower KK scale. At the same time, we expect an enhanced contribution to radiative penguin observables due to the larger number of fermion and boson states in these models, which can circulate in the loop.

The protection mechanism is based on extending the usual SM hypercharge gauge group to a $S U(2)_{R} \times U(1)_{X}$ gauge symmetry in the bulk. ${ }^{20}$ This extended group is then broken to 
$U(1)_{Y}$ on the UV brane. The $U(1)_{Y}$ boson $B_{\mu}$ arises as a linear combination of $X_{\mu}$ and $W_{R, \mu}^{3}$, in analogy to the way the photon is formed from $B_{\mu}$ and $W_{L, \mu}^{3}$ in the SM. The orthogonal linear combination is called $Z_{X}$. Only $B_{\mu}$ has Neumann boundary conditions (BC) on the UV brane; the remaining bosons $\left(Z_{X}, W_{R}^{1,2}\right)$ are endowed with Dirichlet BC. This ensures that only the hypercharge boson has a massless mode. On the IR brane the vector components of all bosons have the ususal Neumann BCs; the Higgs mechanism only breaks $S U(2)_{R} \times S U(2)_{L}$ to the vectorial subgroup and thus large corrections to the $T$-parameter are prevented. To further forbid large corrections to the $Z b b$ vertex from (KK) top quarks an additional discrete $\mathbb{Z}_{2}$ symmetry is usually evoked. ${ }^{30}$ This arranges the quark sector in specific gauge multiplets. ${ }^{31}$ For the lepton sector there is more freedom, as the $Z \tau \tau$ vertex does not necessarily need an additional protection mechanism.

Here, by extension of the SM, we choose the same multiplet structure for leptons and quarks, following Ref. ${ }^{32}$ to which we refer for more details. The lepton sector is built from $\xi_{1 L}$ (bi-doublet) and $\xi_{2 R}$ (singlet) as well as $T_{3}$ and $T_{4}$ which are singlets under $S U(2)_{L}$ and triplets under $S U(2)_{R}$ :

$$
\begin{aligned}
& \xi_{1 L}^{i l}=\left(\begin{array}{cc}
\chi_{L}^{\nu_{i}}(-,+)_{1} & l_{L}^{\nu_{i}}(+,+)_{0} \\
\chi_{L}^{l_{i}}(-,+)_{0} & l_{L}^{l_{i}}(+,+)_{-1}
\end{array}\right) \\
& \xi_{2 R}^{i l}=\nu_{R}(+,+)_{0} \\
& \xi_{3 R}^{i l}=T_{3 R}^{i} \otimes T_{4 R}^{i}=\left(\begin{array}{c}
\tilde{\lambda}_{R}^{i}(-,+)_{1} \\
\tilde{N}_{R}^{i}(-,+)_{0} \\
\tilde{L}_{R}^{i}(-,+)_{-1}
\end{array}\right) \otimes\left(\begin{array}{c}
\lambda_{R}^{i}(-,+)_{1} \\
N_{R}^{i}(-,+)_{0} \\
E_{R}^{i}(+,+)_{-1}
\end{array}\right) .
\end{aligned}
$$

The subscript on the different fermion fields indicates the electric charge $Q$. The signs in parentheses refer to the $\mathrm{BC}$ on the UV (left) and IR brane (right); a "+" corresponds to Neumann and a "-" to Dirichlet BC. This extended fermion sector introduces new topologies for the one loop-diagrams that are not present in the minimal model. The total number of diagrams more than doubles, but only a small subset leads to new integral structures. The basic strategy for the calculation remains the same. As expected, the extended gauge and fermion sector leads to an enhanced gauge-boson contribution of to $g_{\mu}-2:^{14}$

$$
\Delta a_{\mu} \approx 27.2 \cdot 10^{-11} \times\left(\frac{1 \mathrm{TeV}}{T}\right)^{2}
$$

- more than a factor of three larger than for the minimal model. ${ }^{\mathrm{i}}$ The dependence on $T$ and the relative insensitivity of the gauge-boson exchange contributions to 5D masses and Yukawa parameters are general features of RS models with a localized Higgs interactions and do not depend on the precise details of the model.

The Higgs contributions are, in general, model-dependent just as in the minimal case. The main new aspect is the presence of two Yukawa matrices instead of only one. One Yukawa matrix governs the interaction between the bi-doublet and the singlet, while the other one couples the bi-doublet and triplet. The presence of two Yukawa couplings allows for cancellations which make general statements on the size of the total contributions even more difficult.

\footnotetext{
${ }^{i}$ This enhancement would be absent for the simplest lepton multiplet structure, where only the SM singlet is promoted to an $S U(2)_{R}$ doublet.
} 
In particular, if both Yukawas are equal, the dominant contributions cancel, and the Higgs contribution to the dipole operator coefficients becomes negligible.

\section{Summary}

We presented a complete computation of leptonic radiative penguin diagrams in the minimal and custodially protected Randall-Sundrum model. To this end we performed a matching calculation onto $\mathrm{SU}(3) \times \mathrm{SU}(2) \times \mathrm{U}(1)$-invariant dimension-six operators in the unbroken electroweak theory by integrating out the bulk of the warped space-time in a manifestly 5D framework.

Penguin diagrams with gauge bosons in the loop turn out to be technically challenging as the calculation involves up to three bulk coordinate and one 4D loop-momentum integrations. Moreover, while finite, the calculation is only consistent when carried out with a regulator. Without a regularization the sum of short-distance 5D loops and 4D loops with insertions of higher dimensional operators is scheme dependent and cannot be associated with a physical observable.

Surprisingly, the gauge-boson mediated penguin amplitudes are quite insensitive to the 5D Yukawa structure and bulk masses. This allowed us to derive a model-independent result (in the sense of being dependent essentially only on the scale of the model, but not on its other parameters) for their contribution to the muon anomalous magnetic moment.

On the other hand, penguin diagrams that are generated by an internal Higgs boson exchange turn out to be straightforward to compute analytically in dimensional regularization. The main subtlety arises from the sensitivity to the precise implementation of the IR-brane localization of the Higgs. Once this is specified the result is unambiguous, but depends strongly on the parameters of the 5D Lagrangian.

The dimension-six Lagrangian can be utilized to study the consequences of RS models in typical penguin-induced processes like flavour-changing radiative lepton $\ell_{i} \rightarrow \ell_{j} \gamma$ and the magnetic (and electric) dipole moments. We find that the contribution to $g_{\mu}-2$ is enhanced by $\log \frac{k}{T} \approx 35$ compared to the naive dimensional analysis. In the minimal RS set-up the model-parameter independent gauge-boson contribution increases the value of $a_{\mu}$. The effect has the right sign towards the present experimental measurement, but the shift

$$
\Delta a_{\mu} \approx 8.8 \cdot 10^{-11} \times\left(\frac{1 \mathrm{TeV}}{T}\right)^{2}
$$

is too small to resolve the discrepancy of measured value and theory prediction for viable KK masses. ${ }^{j}$ In RS models with an extended bulk gauge and fermion sector like the custodially protected model we generally find larger contributions. In the particular set-up studied in Section 8 the shift is more than a factor of three larger than in the minimal model.

Higgs contributions to the anomalous magnetic moment depend strongly on the size of Yukawa couplings, but are negligible in anarchic models due to the constraints from leptonflavour violating decays. These in turn imply strong restrictions on the Yukawa structure and KK scale, which deserve a more detailed study.

\footnotetext{
${ }^{\mathrm{j}}$ Recall that the lowest KK excitation's mass is around $2.5 T$.
} 


\section{Acknowledgments}

This work has been supported by the DFG SFB/TR 9 "Computergestützte Theoretische Teilchenphysik," the Gottfried Wilhelm Leibniz programme of the Deutsche Forschungsgemeinschaft (DFG), and the DFG cluster of excellence "Origin and Structure of the Universe." The work of JR is supported by STFC UK.

\section{References}

[1] L. Randall and R. Sundrum, Phys.Rev.Lett. 83, 3370 (1999), arXiv:hep-ph/9905221 [hep-ph].

[2] S. Casagrande, F. Goertz, U. Haisch, M. Neubert and T. Pfoh, JHEP 1009, 014 (2010), arXiv:1005.4315 [hep-ph].

[3] A. Azatov, M. Toharia and L. Zhu, Phys.Rev. D82, 056004 (2010), arXiv:1006.5939 [hep-ph].

[4] F. Goertz, U. Haisch and M. Neubert, Phys.Lett. B713, 23 (2012), arXiv:1112.5099 [hep-ph].

[5] M. Carena, S. Casagrande, F. Goertz, U. Haisch and M. Neubert, JHEP 1208, 156 (2012), arXiv:1204.0008 [hep-ph].

[6] R. Malm, M. Neubert, K. Novotny and C. Schmell, JHEP 1401, 173 (2014), arXiv: 1303.5702 [hep-ph].

[7] M. Frank, N. Pourtolami and M. Toharia, Phys.Rev. D89, 016012 (2014), arXiv:1311.1824 [hep-ph].

[8] J. Hahn, C. Hörner, R. Malm, M. Neubert, K. Novotny et al. (2013), arXiv:1312.5731 [hep-ph].

[9] C. Csaki, Y. Grossman, P. Tanedo and Y. Tsai, Phys.Rev. D83, 073002 (2011), arXiv:1004.2037 [hep-ph].

[10] M. Beneke, P. Dey and J. Rohrwild, JHEP 1308, 010 (2013), arXiv:1209.5897 [hep-ph].

[11] O. Gedalia, G. Isidori and G. Perez, Phys.Lett. B682, 200 (2009), arXiv:0905.3264 [hep-ph].

[12] C. Delaunay, J. F. Kamenik, G. Perez and L. Randall, JHEP 1301, 027 (2013), arXiv: 1207.0474 [hep-ph].

[13] M. Blanke, B. Shakya, P. Tanedo and Y. Tsai, JHEP 1208, 038 (2012), arXiv: 1203.6650 [hep-ph].

[14] P. Moch and J. Rohrwild, in preparation.

[15] C. Csaki, A. Falkowski and A. Weiler, JHEP 0809, 008 (2008), arXiv:0804.1954 [hep-ph]. 
[16] M. Blanke, A. J. Buras, B. Duling, S. Gori and A. Weiler, JHEP 0903, 001 (2009), arXiv:0809.1073 [hep-ph].

[17] W. Buchmüller and D. Wyler, Nucl.Phys. B268, 621 (1986).

[18] B. Grzadkowski, M. Iskrzynski, M. Misiak and J. Rosiek, JHEP 1010, 085 (2010), arXiv:1008.4884 [hep-ph].

[19] C. Csaki, J. Erlich and J. Terning, Phys.Rev. D66, 064021 (2002), arXiv:hep-ph/0203034 [hep-ph].

[20] K. Agashe, A. Delgado, M. J. May and R. Sundrum, JHEP 0308, 050 (2003), arXiv:hep-ph/0308036 [hep-ph].

[21] S. Casagrande, F. Goertz, U. Haisch, M. Neubert and T. Pfoh, JHEP 0810, 094 (2008), arXiv:0807.4937 [hep-ph].

[22] A. Höcker and W.J. Marciano, in: Particle Data Book, Phys.Rev. D86, 010001 (2012).

[23] L. Randall and M. D. Schwartz, JHEP 0111, 003 (2001), arXiv:hep-th/0108114 [hep-th].

[24] A. Pomarol, Phys.Lett. B486, 153 (2000), arXiv:hep-ph/9911294 [hep-ph].

[25] Y. Grossman and M. Neubert, Phys.Lett. B474, 361 (2000), arXiv:hep-ph/9912408 [hep-ph].

[26] C. Csaki, C. Grojean, J. Hubisz, Y. Shirman and J. Terning, Phys.Rev. D70, 015012 (2004), arXiv:hep-ph/0310355 [hep-ph].

[27] A. Azatov, M. Toharia and L. Zhu, Phys.Rev. D80, 035016 (2009), arXiv:0906.1990 [hep-ph].

[28] R. Harnik, J. Kopp and J. Zupan, JHEP 1303, 026 (2013), arXiv:1209.1397 [hep-ph].

[29] MEG Collaboration Collaboration (J. Adam et al.), Phys.Rev.Lett. 110, 201801 (2013), arXiv:1303.0754 [hep-ex].

[30] K. Agashe, R. Contino, L. Da Rold and A. Pomarol, Phys.Lett. B641, 62 (2006), arXiv:hep-ph/0605341 [hep-ph].

[31] M. S. Carena, E. Ponton, J. Santiago and C. Wagner, Phys.Rev. D76, 035006 (2007), arXiv:hep-ph/0701055 [hep-ph].

[32] M. E. Albrecht, M. Blanke, A. J. Buras, B. Duling and K. Gemmler, JHEP 0909, 064 (2009), arXiv:0903.2415 [hep-ph]. 